\title{
Nonperturbative real time propagation at finite temperature
}

\author{
E. Mendel * and M. Nest \\ FB Physik, Carl von Ossietzky Universität Oldenburg, 26111 Oldenburg, Germany
}

\begin{abstract}
A new formalism will be presented in order to study real time evolution of quantum systems at finite temperature. Probability distributions for time-correlated observables will be studied non-perturbatively and fully quantized. This works for various systems, including ones with tunneling phenomena. We have obtained good results with some computational methods which can be used on models with several degrees of freedom. Thus it looks feasible to study vacuum tunneling in real time for relevant field theories at finite T.
\end{abstract}

\section{Introduction}

We have been working on a non-perturbative approach to combine real time and finite Temperature for the full quantized theory. This is crucial for the study of systems with tunneling at intermediate temperatures.

We choose to work directly with projectors onto initial and final states, given that the system at finite $T$ is described by the density matrix $\rho$. This method gives a direct probability interpretation for going from states where some degrees of freedom have some values at time 0 , to some other values at time $t$ (in the presence of the thermal bath produced by all the degrees of freedom). This procedure is physically appealing as it multiplies probabilities to be in quantum states (given by $\rho$ ) by the probabilities to go from these states (projected onto some states for the subsystem) to some states at later time. This contrasts with the usual $<\exp (-\beta H)[Q(t)-Q(0)]^{2}>$ to estimate time correlations [1], where the Operator to measure is positive by construction but one has the problem that it is hard to interpret as a transition probability.

The probabilities to find the system in some configuration can be written in a simple way in terms of the path integral formulation in "complex" time. We have been able to solve several systems like the h.osc., the double well, $(1-\cos x)$ potential or the interesting case of a ring of $N$ coupled oscillators, with the aim of the field theory case with vacuum tunneling.

\footnotetext{
${ }^{*}$ Presented at Lattice 97 by E. Mendel.
}

\section{Finite time formalism}

Usually, at finite $T=1 / \beta$, one just takes the trace over Euclidean time $\beta$ to calculate expectation values. For time correlations we will need a path in the complex time plane of the shape: $(0,0) \rightarrow(t,-\epsilon) \rightarrow(0,-2 \epsilon) \rightarrow(0,-\beta)$.

We will combine finite $T$ and real $t$ nonperturbatively, by inserting suitable operators at the points $(0,0),(t,-\epsilon)$ and $(0,-2 \epsilon)$. With a similar path Semenoff, Niemi, Weiss and others]2 deduced Feynman perturbation rules.

To fix ideas, let us ask for the probability that if the first particle (of a system of $N$ interacting quantum particles) is located close to $x_{1}$ at $t=0$, it will be close to $y_{1}$ at time $t$, the system being at $T=1 / \beta$. We will see that this is given by:

$P\left(x_{1}, y_{1}\right)=\int d x_{2} . . d x_{2}^{\prime} . . d y_{2} . . G_{t}\left(x_{1}, x_{2}, . . ; y_{1}, y_{2}, ..\right)$

. $G_{t}^{-1}\left(y_{1}, y_{2}, . . ; x_{1}, x_{2}^{\prime}, ..\right) G_{\beta}\left(x_{1}, x_{2}^{\prime}, . . ; x_{1}, x_{2}, ..\right)(1)$

In order to deduce this equation, we start from the knowledge that for $t=0$ the system is in a mixed state, described by the density matrix:

$\rho=\exp (-\beta H)=\sum_{n} \exp \left(-\beta E_{n}\right)|n><n|$

with the additional condition that the first degree of freedom be at $x_{1}$, which is obtained by applying the projector $P_{x_{1}}:=\left|x_{1}><x_{1}\right| * \mathbb{I}_{2, . . N}$ on both sides of $\rho$. So, for $t=0$ we describe the system by $P_{x_{1}} \rho P_{x_{1}}$. This operator evolves as usual, $U_{t}^{+}\left(P_{x_{1}} \rho P_{x_{1}}\right) U_{t}$, with $U_{t}=\exp (-i H t)$. 
We measure again at a time $t$, with a position $y_{1}$ for the first particle. The probability is then:

$P_{t}^{\beta}\left(x_{1}, y_{1}\right)=\frac{1}{\text { Norm. }} \operatorname{Tr}\left[P_{y_{1}} U_{t}^{+}\left(P_{x_{1}} \rho P_{x_{1}}\right) U_{t}\right]$

where we have discarded one of the $P_{y_{1}}$ due to the cyclicity of the trace. Note that we have to take a small window around $x_{1}$ as otherwise we would measure the position with infinite precision implying total delocalization afterwards (we can see this effect numerically, in that the $P\left(x_{1}, y_{1}\right)$ goes to a constant in $y_{1}$ for very small windows). This can be done easily by defining the projector on a window around the coordinate $x_{1}$ : $\int_{x_{1}-a}^{x_{1}+a} d x_{1}\left|x_{1}><x_{1}\right|$, which still has $P_{x_{1}}^{2}=P_{x_{1}}$. The Normalization can be taken so that the integration over $y_{1}$ in Eq.(3) is one, corresponding to the assumption that the particle $i s$ in the window $\left[x_{1}-a, x_{1}+a\right]$, or with $Z$, in which case we include the a priori probability to be in that window. In both cases the integrated $P$ is $t$-independent.

Notice that classically $\hat{P}_{x_{1}}$ can be commuted with $\hat{\rho}$ so that Eq.(3) would resemble a time correlation among projectors at finite $\beta$, but in the quantum case this ordering gives a probability interpretation, while neglecting one of the $P_{x_{1}}$ one is mixing amplitudes for different states in the thermal mixture, which one should not do...

We can recast Eq.(3) to show that it corresponds to the sum of probabilities to be in states $\mid n>$ with position $x_{1}$ for one of the degrees of freedom by the probability to have gone to $y_{1}$ in time $t$ :

$$
\begin{array}{r}
P\left(x_{1}, y_{1}\right)=\int_{i=2, . ., n} d y_{i} \sum_{n} \exp \left(-\beta E_{n}\right) \\
\cdot\left|\int_{i=2, . ., n} d x_{i} G_{t}\left(y_{1}, y_{i} ; x_{i}, x_{i}\right) \psi_{n}\left(x_{1}, x_{i}\right)\right|^{2}
\end{array}
$$

$\left(G_{t}(x ; y):=<y\left|U_{t}\right| x>\right.$ is the Green function and $\psi_{n}(x)$ a wave function) as we wanted to prove.

Technically it is convenient to rewrite Eq.(3) as

$$
\begin{gathered}
P\left(x_{1}, y_{1}\right)=\int_{i=2, . ., n} d x_{i} d x_{i}^{\prime} d y_{i}<x_{1}, x_{i}|\rho| x_{1}, x_{i}^{\prime}> \\
\cdot<x_{1}, x_{i}^{\prime}\left|U_{t}^{+}\right| y_{1}, y_{i}><y_{1}, y_{i}\left|U_{t}\right| x_{1}, x_{i}>, \quad(5)
\end{gathered}
$$

a product of three Green functions as announced in Eq.(1). The point is that each of these Green functions can be calculated with the method of multiplying the transfer matrix for infinitesimal " $d t$ " by itself $\mathrm{n}$ times to obtain times $t=d t *\left(2^{n}\right)$. This method works even for real $t$ as we showed in our work with Dullweber and Hilf [3]. Alternatively, one can implement a Fast Fourier Transform method [4] to calculate the short time propagator and iterate for longer times. Both of these methods get improved nicely when introducing a small imaginary time $i \epsilon$ in the time propagation, as then the propagators are well behaved at large distances (suppressing the fast oscillations). With this $i \epsilon$ we have even been able to use normal Monte Carlo methods in real time, as now there is some weighting in the sampling, obtaining satisfactory results. The generalization to a field theory seems immediate, but technically it will be hard to implement even on coarse lattices due to the number of degrees of freedom.

\section{Solved models}

We have tested these methods for the systems:

a) for a free particle, the probability to find the particle at some distance $\left|y_{1}-x_{1}\right|$ away has a near to gaussian shape, getting broader with time. As we higher the $T$, the dispersion grows faster.

b) for the harmonic oscillator, as shown in fig.1, from the original window $-.75 \pm .5$ for $t=0$, it oscillates with semiclassical frequency maintaining its coherence (as known for h.osc.). With higher $T$ the packet is broader but stays coherent.

c) the double-well potential shown in fig.2, where the original packet placed at one side disperses through tunneling to the other minima. At higher $T$ the probability to be in the other minima increases, as expected. We are also studying the $(1-\cos (x))^{n}$ potentials with multiple minima to see the dispersion with $t$. This case is relevant, as similar tunneling via instantons in the field theory model is thought to produce the anomaly responsible for baryon asymmetry in the universe.

d) The most interesting case that we have been able to solve, is the $\mathrm{N}$ particle chain of coupled oscillators in a circle, with transversal oscillation. This is the first genuine finite temperature system that we treated in the sense that the Temperature can be dynamically generated by the system in a 
self consistent way (in contrast with an external given heat bath).

We have been able to solve this model "almost" analytically by going to normal modes, $x_{i} \rightarrow X_{s}$. In these new coordinates the Hamiltonian is separable and the N-particle Green function is just

$$
<X_{1}, ., X_{N} ; t\left|X_{1}^{\prime}, ., X_{N}^{\prime} ; 0>=\prod_{s=1}^{N}<X_{s} ; t\right| X_{s}^{\prime} ; 0>\text {. }
$$

The transformation being linear and with Jacobian 1, the Green's function in the original $x_{i}$ is simply equal to the new one, with $X_{s}=X_{s}\left(x_{i}\right)$.

As the integrations in Eq.(1) have to be done numerically we have been able to solve for up to $N=4$ particles. The probability distribution is again some Gaussian-like curve that gets broader with time (the zero mode corresponding to a collective movement of the circle of strings is allowed and so the center of mass of the system disperses as usual in QM). As we increase the temperature the coordinate $x_{1}$ disperses much faster due to the momentum transfer from the other oscillators. It would be interesting in the future to study to which extent we have reached the thermodynamic limit in the sense that if we have a very large circle of $N$ oscillators the probability to find the first in some position after some time, should converge to some limit which should not change when we go to $N+1$ oscillators.

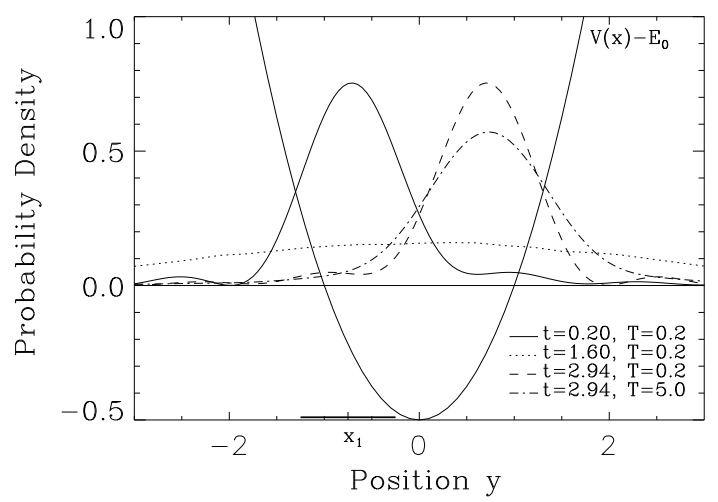

Figure 1. Probability to be at $y$ for an h.osc. for times $t \approx 0, \pi / 2$ and $\pi$, at low and high $T$.

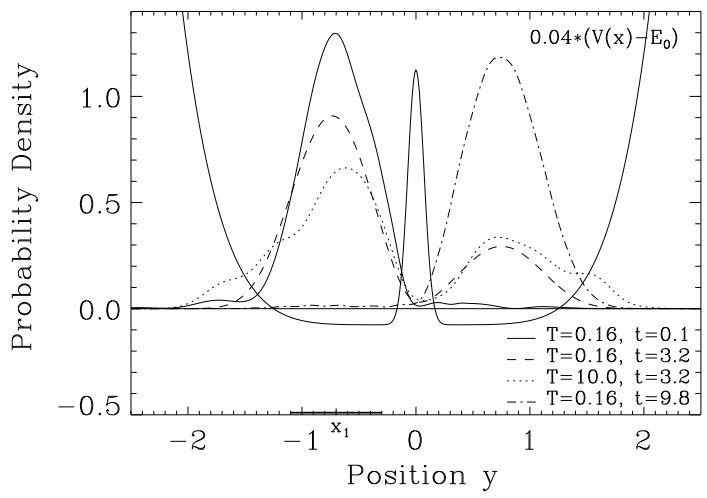

Figure 2. Probability to be at $y$ for a double well for $t \approx 0, .3$ and 1 . of tunneling time, for two $T$.

\section{Conclusions}

We have been able to combine non perturbatively real time and finite Temperature in first quantization. It can be seen as the product of three Green functions in the complex time plane. The "matrix iteration" and the FFT methods work well in calculating the real time path integral, improving convergence with a small $i \epsilon$.

There are several interesting problems that seem shortly doable, like the ammonia molecule or several spin models (easy due to discrete field values). Our ultimate aim is to find a way to treat the field theory case of tunneling with instantons in real time and finite $T$, which is in principle doable with these methods but will be hard to implement due to the number of degrees of freedom.

We thank L. Polley for useful discussions and the RRZN in Hannover for supercomputer time.

\section{REFERENCES}

1. G. Aarts and J. Smit, Phys. Lett. B393 (1997) 395 and refs. therein; J.Ambjørn and A.Krasnitz, Phys. Lett. B362 (1995) 97.

2. R.Kobes, G.Semenoff and N.Weiss, Z. Phys. C29 (1985) 371 and refs. therein.

3. A.Dullweber, E.Hilf and E.Mendel, quant-ph: 9511042; E. Mendel and M. Nest, in prepar.

4. E.Onofri and G.Tecchiolli, Phys. Scripta 37 
(1988) 323 and refs. therein. 